\title{
EFFECT OF A NOVEL CERAMIC SURFACE TREATMENT ON THE RETENTION OF LITHIUM DISILICATE CROWNS BONDED WITH TWO DIFFERENT LUTING APPROACHES AN INVITRO STUDY
}

\author{
Moataz Mostafa Anwar ${ }^{*}$, Adel ElTannir ${ }^{* *}$ and Reham ElBasty***
}

\begin{abstract}
Aim: To evaluate the effect of the novel one step ceramic surface treatment on the retention of lithium disilicate crowns cemented with two different luting approaches (self-etch and selfadhesive resin cements) compared to the conventional surface treatment technique.

Materials and methods: 28 extracted molar teeth were prepared to receive full coverage lithium disilicate glass ceramic crowns with lateral projections for pull out test. The crowns were divided into 2 main groups according to the surface treatment applied, Group 1: conventional surface treatment by hydrofluoric acid followed by silane coupling agent (HF+silane), Group 2: the new self-etching ceramic primer Monobond etch and prime(MEP). Each group was subdivided into 2 subgroups according to cementation protocol: In Subgroup A crowns were bonded using self- etch resin cement (Rely X Ultimate) while in subgroup B, crowns were bonded using selfadhesive resin cements (Rely X U200). Crowns were tested for retention using pull out test until failure occurred.
\end{abstract}

Results: Group 2 showed statistically insignificant higher retention mean value (204.63 $35.30 \mathrm{~N})$ than Group $1(196.21 \pm 39.76 \mathrm{~N})$, while subgroup A $(265.75 \pm 49.35 \mathrm{~N})$ showed statistically significant higher retention mean value than subgroup B $(135.09 \pm 25.71 \mathrm{~N})$. Subgroup $2 \mathrm{~A}$ (MEP and Rely X Ultimate) had the highest bond strength $(267.42 \pm 40.92 \mathrm{~N})$ followed by $1 \mathrm{~A}(\mathrm{HF}+$ silane and Rely X Ultimate), 2B (MEP and Rely X U200), 1B(HF+ silane and Rely X U200).

Conclusion: Self-etching ceramic primer MEP is strongly recommended to replace conventional surface treatment. Self-etching ceramic primer (MEP) with self-etch resin cement provided the highest bond strength.

\footnotetext{
* Fixed Prosthodontics, Faculty of Dentistry, Cairo University, Cairo, Egypt

** Department of Fixed Prosthodontics, Faculty of Dentistry, Cairo University, Cairo, Egypt
} 


\section{INTRODUCTION}

Glass ceramics are widely used in the field of restorative dentistry to replace the hard dental tissue. This is due to the fact that they are highly durable and that they provide excellent esthetic quality (Höland et al. 2006).

Lithium disilicate reinforced glass ceramics are available in the dental field since 1998. In 2009, the pressable ingot (IPS e.max press, Ivoclar Viva dent) (flexural strength: $400 \pm 40 \mathrm{MPa}$ ) which is composed of approximately $70 \%$ lithium disilicate crystals by volume. The crystals' size (3-6um) and their uniform distribution among the glass matrix made up the favorable mechanical and optical properties of the material. The ingots are available in different shades which can be characterized by a simple staining technique. This allowed the clinicians to provide monolithic esthetic and functional restorations. In addition, this omitted the error of porcelain veneer chipping error especially in the posterior area thus increasing the longevity of the restoration (Zarone et al. 2016) ("The Original Lithium Disilicate Press Ceramic The Legendary Press Ceramic," n.d.).

Producing efficient bonding to glass ceramics is essential to improve the strength and durability of the restoration. To provide micromechanical and chemical means of bonding, the restorations' fitting surfaces are treated by the conventional technique (HF acid followed by the application of silane coupling agent). Hydrofluoric acid application results in alteration of the surface topography by the production of micro and nanoporosities that differ in width and depth which increases the surface area for bonding to resin composites. In addition, HF acid increases the surface energy of the etched surface to improve the wettability of the hydrophobic silane coupling agent (Ramakrishnaiah et al. 2016).

The silane coupling agent is a bifunctional monomer that produces bonding between the inorganic phase of ceramic (by acting on the silica particles forming a siloxane group) and the organic phase of the adhesive resin cement. In addition, it increases wettability of the cement to the surface of the glass ceramic by increasing the surface energy of the ceramic substrate (Baratto et al. 2015).

However, the conventional approach for treating glass ceramics has several drawbacks. It is technique sensitive and time consuming in addition to the inherent toxicity of the HF acid. The novel 1 step self-etching glass ceramic primer "Monobond etch and prime" (Ivoclar Vivadent) was introduced to the dental market in 2015 as an alternative for the previously described conventional surface treatment of lithium disilicate ceramics. It contains a trimethoxypropyl methacrylate for silanization and a new polyfluoride for the etching step which eliminates the need for using the toxic HF acid. The efficiency of this material needs further investigation ("Scientific Documentation - PDF" n.d.).

There are two luting protocols for cementing lithium disilicate glass ceramic restorations. The first is using a self-etch resin cement, which needs tooth preparation for adhesion (by etching of enamel using phosphoric acid followed by application of a self-etch/multipurpose adhesive to etch dentinal tissue and prime both enamel and dentin). The second approach is through using self-adhesive resin cements which do not need any preceding intervention applied to the tooth. They are composed of multifunctional acid methacrylate, the acidic content causes demineralization of dentin, while the methacrylate component(resin) infiltrates dentin forming hybrid layer and resin tags, with the smear layer incorporated in the hybrid layer (Moghaddas et al. 2017).

The highest possible bond strength is the ultimate goal of restorative intervention. Finding out the appropriate combination between the suitable ceramic surface treatment with the reliable cementation protocol needs to be figured out to reach 
durable bond strength to both the lithium disilicate restoration and the tooth substrate. Therefore, the aim of this study was to evaluate the effect of the novel one step ceramic surface treatment on the retention of lithium disilicate crowns cemented with two different luting approaches (self-etch and self-adhesive resin cements) compared to the conventional surface treatment technique. The first hypothesis of this research states that the novel one step ceramic surface treatment will give the same results as the conventional treatment technique. The second hypothesis states that both cementation approaches will give similar results.

\section{MATERIALS}

\begin{tabular}{|c|c|}
\hline $\begin{array}{c}\text { IPS Emax press } \\
\text { ingots }\end{array}$ & $\begin{array}{c}\text { Ivoclar Vivadent, Schann, Liechtenstein, } \\
\text { Germany. }\end{array}$ \\
\hline $\begin{array}{c}\text { IPS Ceramic } \\
\text { etching gel }\end{array}$ & $\begin{array}{c}\text { Ivoclar Vivadent, Schann, Liechtenstein, } \\
\text { Germany. }\end{array}$ \\
\hline $\begin{array}{c}\text { Monobond etch } \\
\text { and prime }\end{array}$ & $\begin{array}{c}\text { Ivoclar Vivadent, Schann, Liechtenstein, } \\
\text { Germany. }\end{array}$ \\
\hline $\begin{array}{c}\text { Rely X } \\
\text { Ceramic primer }\end{array}$ & $\begin{array}{c}\text { 3M Deutschland GmbH, Dental } \\
\text { Products, Neuss, Germany. }\end{array}$ \\
\hline $\begin{array}{c}\text { Scotchbond } \\
\text { Universal adhesive }\end{array}$ & $\begin{array}{c}\text { 3M Deutschland GmbH, Dental } \\
\text { Products, Neuss, Germany. }\end{array}$ \\
\hline $\begin{array}{c}\text { Rely X Ultimate } \\
\text { resin cement }\end{array}$ & $\begin{array}{c}\text { 3M Deutschland GmbH, Dental } \\
\text { Products, Neuss, Germany. }\end{array}$ \\
\hline $\begin{array}{c}\text { Rely X U200 } \\
\text { resin cement }\end{array}$ & $\begin{array}{r}\text { 3M Deutschland GmbH, Dental } \\
\text { Products, Neuss, Germany. }\end{array}$ \\
\hline
\end{tabular}

\section{METHODS}

\section{1) Teeth Selection and grouping}

28 extracted intact upper molar teeth were selected. Teeth were disinfected using 1:10 sodium hypochlorite solution and stored in $0.9 \%$ saline solution. They were then mounted in epoxy resin molds using a paralleling device. Measurements of the mesiodistal and buccolingual dimensions were taken using a digital caliper. It was ensured that the teeth had close measurements to standardize the bonding surface area of the teeth substrate. Preparation of the teeth was done to receive pressed lithium disilicate full coverage crowns according to the manufacturer's instructions. Silicone indicies for teeth before preparation were fabricated and sectioned in a buccolingual direction. Occlusal preparation was done (using blue grit tapered stone with rounded end*) to reach $1.5 \mathrm{~mm}$ preparation depth on both functional and nonfunctional cusps as recommended by Ivoclar Vivadent. The amount of occlusal preparation was verified by the silicon index and a graduated probe.

Axial preparation was done with blue grit tapered stone with rounded end to obtain deep chamfer finish line $1 \mathrm{~mm}$ in width. Preparation followed the anatomy of the teeth. Axial surfaces were prepared using a milling machine with an attached straight hand piece (AF30 milling machine, Nouvag, Switzerland to ensure standardization of the convergence angle (15 degrees) (Johnson et al. 2014).

A silicone index was also used to ensure the amount of axial preparation on each surface.

Intermittent water cooling was applied to avoid heat generation and desiccation of dentin. Polishing of the prepared teeth was performed using a cylindrical polishing rubber with rounded end of red grit (Prep twins, Intensiv Inc.).

\section{2) Construction of IPS e-max press crowns:}

Prepared teeth were scanned using an extra oral scanner (Neway extraoral scanner, Open technologies, Faro technologies Inc.) to produce virtual dies. The full coverage crowns were designed using a design software (Exocad 2.2 Valleta).

Two horizontal bars were designed proximally to allow engagement of the crowns in the pull out test (Johnson et al. 2014) 


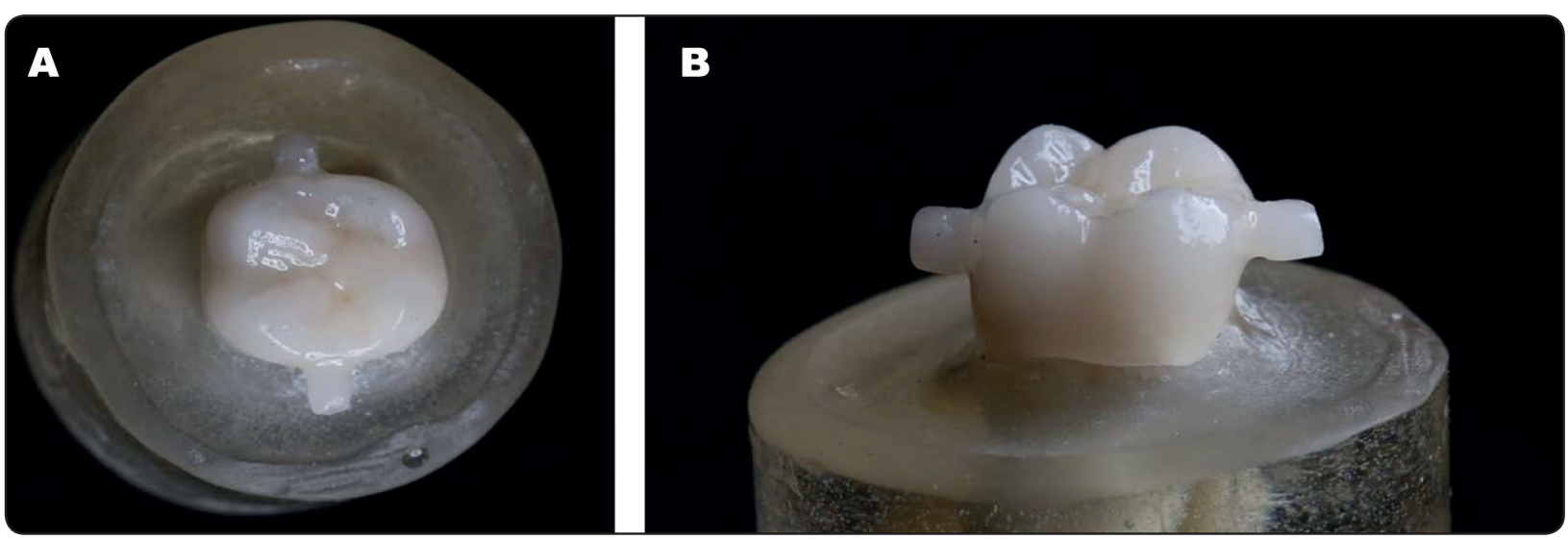

Fig. (1): IPS e.max press crowns after finishing and glazing (A) Occlusal view (B) Buccal view

After designing the crowns on the CAD software, they were milled using CAD wax blanks. Crowns were then ready for pressing using LT e-max ingots (shade A1) (Ivoclar Vivadent, Schaan, Liechtenstein, Germany).

Spruing, investing and wax burnout was done according to the manufacturer's instructions. Pressing was done utilizing the pressing furnace (Programmat LP3010 pressing furnace, Ivoclar Vivadent, Schaan, Liechtenstein, Germany) through the IPS e.max pressing program). After the pressing program was completed the investment ring was left to cool to room temperature, divesting procedure followed according to the manufacturer's instructions. Crowns were divested (through rough divesting by medium polishing jet at a pressure of 4 bars) until the crowns were visible then fine divestment at 2 bar was applied. Reaction layer was removed; crowns were finished according to manufacturer's instructions. Glazing of the crowns was done according to manufacturer's instructions Figure (1).

\section{3) Teeth groupimg:}

Lithium disilicate crowns $(\mathbf{n = 2 8})$ were divided into 2 main groups Figure (2) according to the surface treatment protocol. In The first group (n=14), the surface treatment used was applying ceramic etching gel (5\% hydrofluoric acid) to the fitting surface of the crowns followed by silane application "Control group" (Group 1: HF+ silane). In The second group $(\mathbf{n}=14)$ the surface treatment used was applying the novel self-etching ceramic primer (Monobond etch and prime) "Intervention group" (Group 2: MEP).

Each group was further divided into 2 subgroups according to the type of adhesive resin cement used. In Subgroup $A(n=7)$ : Self etch resin cement was used (Rely X Ultimate clicker) while in Subgroup B $(n=7)$ : Self-adhesive resin cement was used (Rely X U200) Figure (2).

\section{4) Bonding of IPS e-max press crowns:}

\section{A) Surface treatment of the crowns:}

\section{- Group 1: $(\mathrm{HF}+$ silane $)$}

IPS e.max press crowns were first treated by IPS Ceramic etching gel (5\% HF acid). It was applied on the fitting surfaces of the crowns using a disposable brush and left in contact with the surface for 20 secs as recommended by Ivoclar Vivadent . Afterwards, it was thoroughly rinsed under running water then dried thoroughly with oil free air.

In order to remove the residues of the reaction, active application of $37 \%$ phosphoric acid then follows. The acid was placed on the fitting surface 


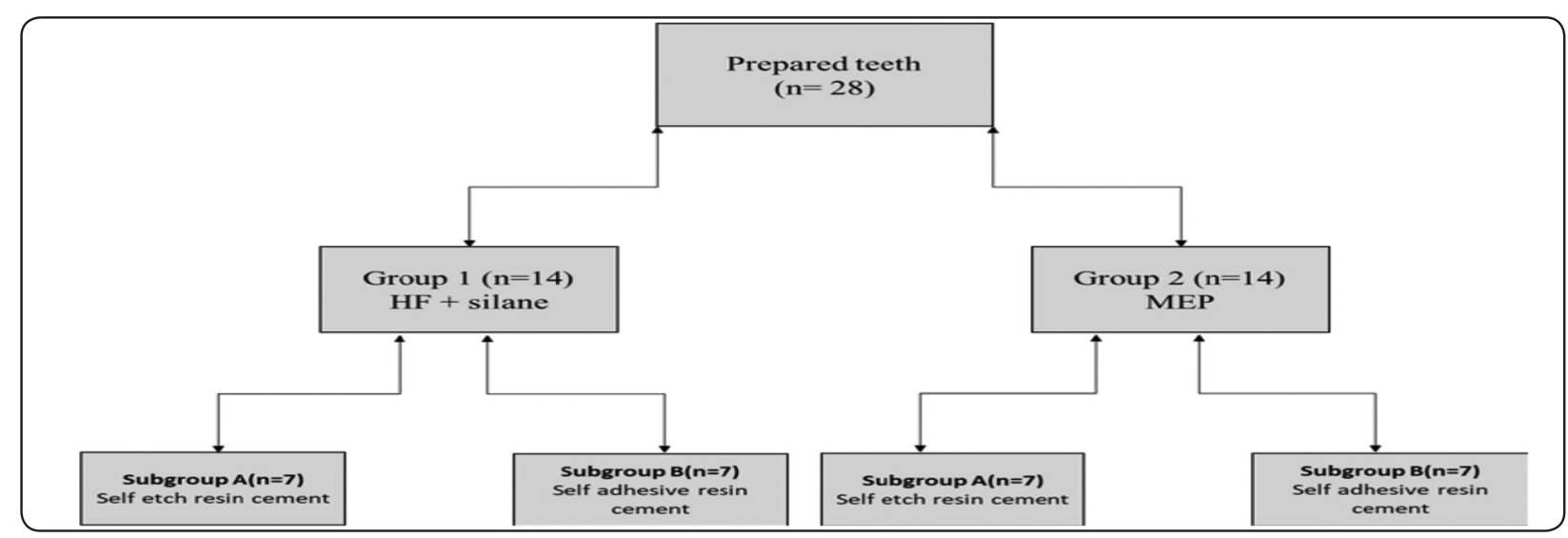

Fig. (2): Schematic presentation of the main groups and subgroups

of the restoration and agitated for 30 secs then rinsed away under running water and dried thoroughly with oil free air.

The silane coupling agent was then applied, (Rely X ceramic primer, 3M Deutschland GmbH, Dental Products, Neuss, Germany), once on the etched crowns surfaces according to manufacturer's instructions using a microbrush. It was left to react for 60 secs. Oil free air was blown gently to allow excess solvent to evaporate.

\section{- Group 2: (MEP)}

IPS e.max press crowns were treated using the self-etching ceramic primer (Monobond Etch and Prime (MEP)* Figure (3). With reference to manufacturer's instructions, MEP was applied to the fitting surfaces of the e-max press crowns with a microbrush, rubbed for $20 \mathrm{secs}$, left on the ceramic surface to react for 40 secs then it was washed thoroughly with water (until the green color of MEP is not seen) and dried with oil free air for 10 seconds.

\section{B) Surface treatment of the tooth substrate}

\section{- For Subgroup A (self-etch resin cement, Rely X Ultimate)}

The prepared teeth were rinsed with water followed by drying with oil free air. Since the preparation was totally within dentin, universal adhesive (Scotchbond Universal, 3M Deutschland $\mathrm{GmbH}$, Dental Products, Neuss, Germany) was applied to the prepared teeth using a microbrush and scrubbed for 20 secs, air thinned with a gentle stream of oil free air for 5 seconds and left without curing. A thin layer of the universal adhesive was applied to the fitting surface of the crown according to the manufacturer's instructions, thinned with a gentle stream of oil free air and left without curing

On a mixing pad, equal amounts (1:1) of base and catalyst pastes of the resin cement (Rely X Ultimate clicker, 3M Deutschland GmbH, Dental Products, Neuss, Germany) were dispensed (by applying 2 clicks) and mixed with a plastic spatula. The mix was applied axially on the fitting surface of the crowns.

The crowns were seated first with finger pressure on the tooth. Tack curing for $1 \mathrm{sec}$ was done where the excess cement reached a rubber like consistency that facilitated its removal using a manual scaler.

A load of 5 kilograms was applied axially on the crowns through placing it in a cementing device. The selected weight is then placed on a piston that is applied perpendicular to the occlusal surface of the crown and along its long axis keeping it under compressive load until complete curing of the 
cement). Final curing was applied for 20 seconds (using a light curing unit of $1200 \mathrm{~mW} / \mathrm{cm}^{2}$, Elipar S10) to each surface after applying oxyguard (from Panavia F kit, Kurary Noritake) to the margins of the crown. Any remaining excess cement was removed with a finishing stone. The $5 \mathrm{~kg}$ load was left on the crown for 10 minutes until complete curing of the cement. The process was repeated for the crowns of the same subgroup.

\section{- For Subgroup B (self-adhesive resin cement, Rely X U200):}

The prepared teeth were rinsed with water and dried with oil free air without any further treatment. On a mixing pad, equal amount (1:1) of base and catalyst pastes of the self-adhesive resin cement (Rely X U200 clicker, 3M Deutschland $\mathrm{GmbH}$, Dental Products, Neuss, Germany) were dispensed (by applying 2 clicks) and mixed with a plastic spatula. The mix was applied in the fitting surface of the crowns which were seated on their corresponding prepared teeth using the cementing device. Removal of excess cement and final curing were done as done previously.

\section{Pull out test}

Retention was measured using Materials Testing Machine (Model 3345; Instron Industrial Products, Norwood, USA) using a load cell of $5 \mathrm{kN}$ (Figure 3). Data were recorded using computer

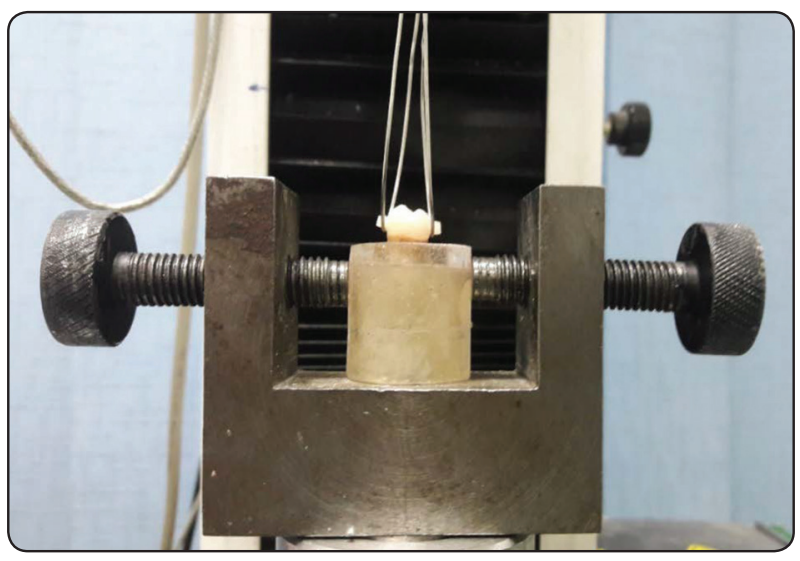

Fig. (3): Pull out test software (Bluehill Lite; Instron Instruments). The crown was suspended from the upper movable compartment of the testing machine by double orthodontic wire loop $(0.7 \mathrm{~mm})$ through the lateral projections ( 2 horizontal bars on the proximal surface) made during milling. The device was subjected to a slowly increasing vertical load $(1 \mathrm{~mm} /$ min) until failure (whether cohesive, adhesive or mixed). The load required to dislodgment was recorded in Newtons.

\subsection{Statistical analysis:}

The results were analyzed using Graph Pad Instat (Graph Pad, Inc.) software for windows. A value of $\mathrm{P} \leq 0.05$ was considered statistically significant. Continuous variables were expressed as the mean and standard deviation. After homogeneity of variance and normal distribution of errors had been confirmed, student t-test was done for compared pairs. Two-way ANOVA to show effect of variables (main groups and subgroups). Chi square test was done between failure modes score. Sample size $(n=7)$ was large enough to detect large effect sizes for main effects and pair-wise comparisons, with the satisfactory level of power set at $80 \%$ and a $95 \%$ confidence level.

\section{RESULTS}

\section{Retention}

Descriptive statistics of retention $(\mathrm{N})$ showing mean, standard deviation (SD), minimum, maximum and $95 \%$ confidence intervals (CI) (low and high) values for both groups as function of cementation approach is summarized in Table (1) and graphically drawn in Figure (4).

\section{1) Total effect of main groups (Group 1 vs. group 2)}

Regardless of other variables, it was found that Group 2 recorded statistically non-significant higher retention mean value $(204.63 \pm 35.30 \mathrm{~N})$ than Group 1 mean value $(196.21 \pm 39.76 \mathrm{~N})$ as indicated by two-way ANOVA test ( $\mathrm{p}$ value $=0.5314>0.05$ ). 
TABLE (1): Descriptive statistics of retention results (Mean \pm SD) for both groups as function of cementation approach.

\begin{tabular}{|c|c|c|c|c|c|c|c|c|}
\hline \multirow{2}{*}{\multicolumn{2}{|c|}{ Variable }} & \multirow{2}{*}{ Mean } & \multirow{2}{*}{ $\pm S D$} & \multicolumn{2}{|c|}{ Range } & \multicolumn{2}{|c|}{$95 \% C I$} & \multirow{2}{*}{$\frac{\text { t-test }}{P \text { value }}$} \\
\hline & & & & Minim. & Maxim. & Lower & Upper & \\
\hline \multirow[b]{2}{*}{ Group 1} & Subgr_1_A & 264.07 & 57.78 & 207.79 & 379.63 & 215.76 & 312.38 & \multirow{2}{*}{$<0.0001 *$} \\
\hline & Subgr_1_B & 128.35 & 21.75 & 96.07 & 161.10 & 110.17 & 146.53 & \\
\hline \multirow[b]{2}{*}{ Group 2} & Subgr_2_A & 267.42 & 40.92 & 239.81 & 321.64 & 233.21 & 301.63 & \multirow{2}{*}{$<0.0001 *$} \\
\hline & Subgr_2_B & 141.84 & 29.68 & 98.61 & 185.50 & 117.02 & 166.65 & \\
\hline
\end{tabular}

*; significant $(p<0.05)$

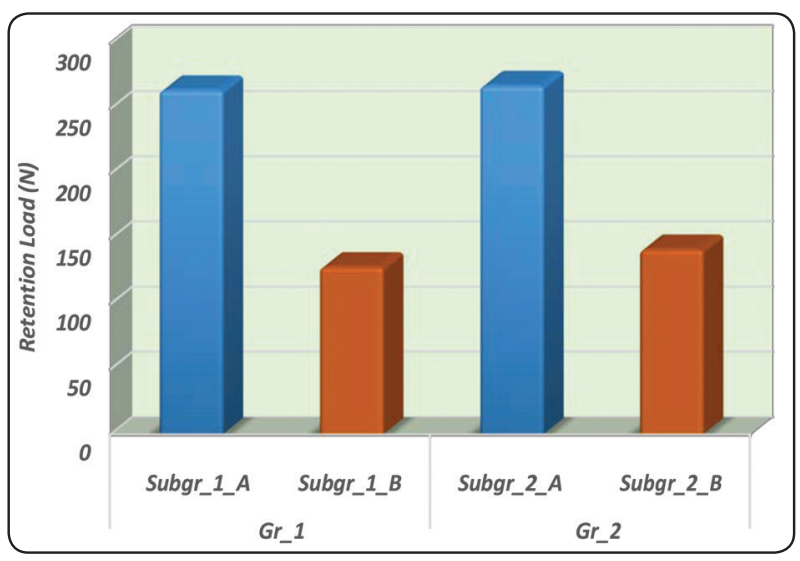

Fig. (4): Column chart showing retention results mean values for both lased groups as function of cementation approach.

In Group 1; it was found that the retention mean \pm SD values recorded for subgroup 1A (subgr_1_A) ( (HF + silane with Rely X Ultimate) were (264.07 $\pm 57.78 \mathrm{~N})$ with minimum value $(207.79 \mathrm{~N})$ and maximum value $(379.63 \mathrm{~N})$.

Meanwhile the retention mean $\pm \mathrm{SD}$ values recorded for subgroup 1B (subgr_1_B) $((\mathrm{HF}+$ silane with Rely X U200) group were (128.35 \pm 21.75 $\mathrm{N})$ with minimum value $(96.07 \mathrm{~N})$ and maximum value $(161.10 \mathrm{~N})$. It was found that $s u b g r_{-} \boldsymbol{1}_{-} \boldsymbol{A}$ recorded statistically significant $(\mathrm{p}<0.05)$ higher mean value than subgr_1_B mean value as indicated by paired t-test. Table (1): Descriptive statistics of retention results (Mean $\pm S D)$ for both groups as function of cementation approach. In
Group 2; it was found that the retention mean $\pm \mathrm{SD}$ values recorded for Subgroup 2A(subgr_2_A)( (MEP with Rely X Ultimate) were (267.42 \pm 40.92 $\mathrm{N})$ with minimum value $(239.81 \mathrm{~N})$ and maximum value $(321.64 \mathrm{~N})$

Meanwhile the retention mean \pm SD values recorded for Subgroup 2B (subgr_2_B) ((MEP with Rely X U200) group were $(141.84 \pm 29.68 \mathrm{~N})$ with minimum value $(98.61 \mathrm{~N})$ and maximum value $(185.50 \mathrm{~N})$. It was found that $s u b g r \_2 \_A$ recorded statistically significant $(\mathbf{p}<0.05)$ higher mean value than $\boldsymbol{s u b g r} \_2 \_B$ mean value as designated by paired t-test.

In $s u b g r \_A$; it was found that subgr $2 \_A$ recorded statistically non-significant higher mean value $(267.42 \pm 40.92 \mathrm{~N})$ than subgr_1_A one $(264.07 \pm 57.78 \mathrm{~N})$ as proven by unpaired t-test (p $=0.8954>0.05$ )

In $s u b g r \_B$; it was found that subgr_2_B recorded statistically non-significant higher mean value $(141.84 \pm 29.68 \mathrm{~N})$ than $\boldsymbol{s u b g r} \_$1_B $(128.35 \pm 21.75 \mathrm{~N})$ as verified by unpaired t-test $(\mathrm{p}=$ $0.3175>0.05)$

\section{2) Total effect of subgroups (subgr_A vs. subgr_B)}

Regardless of other variables, it was found that subgr_A recorded statistically significant higher retention mean value $(265.75 \pm 49.35 \mathrm{~N})$ than subgr_B mean value $(135.09 \pm 25.71 \mathrm{~N})$ as indicated by two-way ANOVA test ( $\mathrm{p}$ value $=<0.0001<0.05$ ). 


\section{3) Modes of failure}

Observing modes of failure is also an indicative of the bond strength, subgroup $1 A(H F+$ silane with Rely X Ulimate) and $2 A$ (MEP with Rely X Ultimate) showed cohesive mode of failure within the IPS e.max press crowns. On the contrary, subgroup $1 B$ (HF + silane with Rely X U200) showed adhesive mode of failure between the cement and the tooth substrate. One sample of group $2 B$ (MEP with Rely $X$ U200) showed mixed adhesive cohesive mode of failure while the rest of the subgroup showed adhesive mode of failure between the cement and the tooth substrate.

Frequent distribution of failure modes scores (\%) for all subgroups after are summarized in Table (2) Stacked column chart showing frequent distribution of failure modes scores (\%) for all subgroups). The difference in frequent distribution of failure modes scores between subgroups was statistically significant as indicated by chi square test $(\mathrm{P}=<0.0001<0.05)$

TABLE (2): Comparison of frequent distribution for failure modes scores (\%) between all subgroups

\begin{tabular}{|c|c|c|c|c|c|c|}
\hline & \multirow[b]{2}{*}{ Variable } & \multicolumn{3}{|c|}{ Failure modes } & \multicolumn{2}{|c|}{ Chi square test } \\
\hline & & Adhesive & Cohesive & Mixed & Chi value & $\mathrm{P}$ value \\
\hline \multirow{2}{*}{$\vec{J}$} & Subgr_1_A & 0 & 100 & 0 & \multirow{4}{*}{430.11} & \multirow{4}{*}{$\begin{array}{l}\frac{*}{\delta} \\
\stackrel{8}{8} \\
\dot{\nabla} \\
\text { v }\end{array}$} \\
\hline & Subgr_1_B & 100 & 0 & 0 & & \\
\hline \multirow{2}{*}{$\begin{array}{l}{ }_{1} \\
\dot{3}\end{array}$} & Subgr_2_A & 0 & 100 & 0 & & \\
\hline & Subgr_2_B & 85.7 & 0 & 14.3 & & \\
\hline
\end{tabular}

\section{DISCUSSION}

Glass ceramics are considered an essential component of esthetic dentistry. Their favorable mechanical properties, biocompatibility, inertness, wear resistance in addition to excellent esthetic properties made them the favorable choice of many clinicians (Pollington 2011).
The fitting surface of the adhesive glass ceramics should be treated in order to strengthen the bond with the adhesive resin cement (Brum et al. 2011). The conventional surface treatment for glass ceramics involves the use of hydrofluoric acid (HF acid) followed by silane coupling agent application. However, HF acid can cause burns to the skin owing to its chemical toxicity. It is considered a health hazard to both clinicians and dental personnel.

Monobond etch and prime (MEP, Ivoclar Vivadent) is a self-etching ceramic primer that omits the use of hydrofluoric acid. There is insufficient research considering the effectiveness of this product compared to the conventional treatment. Most studies favored the conventional approach in terms of bond strength, but MEP demonstrated comparable results ("Scientific Documentation PDF" n.d.).

Glass ceramics should be better cemented with resin cements to allow for a strong bond with the tooth substrate. Resin cements are classified into total etch, self-etch or self-adhesive according to the composition and bonding technique. Self-etch resin cements require the use of a self-etching primer to treat the tooth substrate. While the selfadhesive resin cements do not require any tooth pretreatment therefore they are considered easier to use. (Stamatacos and Simon 2013).

Therefore, the aim of this study was to evaluate the effect of the novel one step ceramic surface treatment on the retention of lithium disilicate crowns cemented with two different luting approaches (selfetch and self-adhesive resin cements) compared to the conventional surface treatment technique.

Extracted natural teeth were selected because they are considered the best substrates to test bond strength over any other materials. As stated by Nawrocka and Lukomska-Szymańska 2019, the dental tissues are considered "irreplaceable in laboratory tests". The properties of the natural enamel and dentin cannot be substituted by artificial materials. In addition, natural teeth were selected 
to simulate the clinical workflow. Molars were selected as they have larger surface area available for bonding compared to premolars and anteriors.

Extracted molars were mounted in epoxy resin molds to ease their handling and allow seating on the milling machine plate. This was done using a paralleling device to ensure seating of the teeth perpendicular to the horizontal plane of the epoxy resin molds and avoid angulation or tilting of the teeth. Epoxy resin margin was terminated $2 \mathrm{~mm}$ below cementoenamel junction to allow its visualization.

Tooth preparation was done using a milling machine to standardize the convergence angle of all specimens (Johnson et al. 2014). Silicone indices were also made to ensure standardization of the amount of tooth preparation.

In this study, lithium disilicate reinforced glass ceramic was used as it is the most widely used glass ceramic. It is considered a gold standard due to its reliable adhesive, mechanical and esthetic properties (either in a monolithic or layered form) (Qamheya AHA 2016). This was approved by multiple researches throughout the past decade. IPS e-max press ingots were used as the lithium disilicate reinforced glass ceramic. The pressable ceramic was used rather than milled as the to facilitate generation of the 2 horizontal proximal bars needed for the pull out test. This was applying by designing the horzintal bars on the software and using CAD wax for fabrication of the wax patterns needed for pressing. In addition, the pressable ceramic has higher strength, better marginal adaptation and decreased surface defects (Paper, n.d.).

IPS e.max press low translucency ingots were used as recommended by Ivoclar Vivadent company for fabrication of posterior crowns (Vivadent 2012). A1 shade was used to ensure the best light transmission through the crowns to the underlying resin cement in order to ensure proper depth of curing of the resin cement (Peixoto et al. 2007). Spruing, investing, pressing, finishing and glazing was done according to the manufacturer's instructions to follow recommendations of the manufacturing company (Vivadent 2012).

The fitting surfaces of Group 1 crowns were subjected to HF acid treatment followed by silane coupling agent ( $\mathrm{HF}+$ silane) which is considered the gold standard for surface treatment of glass ceramics. HF acid was left in contact with the ceramic surface for 20 secs as recommended by the manufacturer's instructions to produce its effect by reacting with silica of the glassy matrix producing fluorosilicilic acid. This compound is easily washed away by rinsing with water and hence the next step was rinsing the fitting surfaces under running water to remove excess HF acid as well as flurosilicilic acid. The reaction leads to formation of microporosities in the glassy matrix and increases its surface energy.

Active application of phosphoric acid then followed for 30 secs to remove residues of the reaction by agitation of the acid with a microbrush to produce better efficiency in removing reaction residues of HF with glass ceramics as suggested by (Giraldo et al. 2016) .

After rinsing of phosphoric acid and dryness, silane coupling agent was then applied to react with active ceramic surface were it bonds to silica particles through alkoxy group. It is left for 60 secs to allow bonding to silica and the methacrylate group remains active and ready to bond with resin cement monomers. Gentle blowing with oil free air allows excess alcohol solvent to evaporate.

As for Group 2 crowns, surface treatment by selfetching ceramic primer was done as an alternative to the conventional surface treatment. Monobond etch and prime (MEP) was applied on the fitting surface of the crown, rubbed for 20 secs then left for 40 secs were ammonium polyfluoride reacts with ceramic surface. The reaction roughens and activates the surface. The silane within MEP then chemically bonds to the activated surface. Rinsing followed, to remove the excess ammonium polyfluoride, and then silicon oxygen bond was formed. By completely drying the surface for 10 secs, condensation of the 
silane onto the surface occurs forming a stable bond with free methacrylate groups to bond with adhesive resin cement ("Scientific Documentation PDF” n.d.) (Prado et al. 2018).

The 2 main groups were further subdivided into 2 subgroups according to the type of adhesive resin cements (either self-etch or self-adhesive resin cements). This was applied to test 2 different techniques of bonding glass ceramics which are clinically acceptable.

In Subgroup A, crowns were cemented using self-etch resin cement. As the preparation was solely in dentin, it was treated using a self-etching primer having acidic monomers that etch and prime dentin plus the presence of 10-MDP monomer that bond chemically to calcium content of dentinal hydroxyapatite crystals (Carrilho et al. 2019). The use of phosphoric acid for etching dentinal tissue was omitted as it causes desiccation of collagen bundles, decreases bond strength overtime and clinically causes postoperative hypersensitivity (Betancourt, Baldion, and Castellanos 2019). Oxyguard was applied to the margins to avoid oxygen inhibited layer.

While in Subgroup B, crowns were cemented using self-adhesive resin cements which did not require any pretreatment of the tooth. This is because it contains acidic monomers that are able to etch and prime dentinal tissues simultaneously.

Retention was measured using pull out test in order to simulate clinical conditions that could occur during mastication. 0.7 orthodontic wire thickness was used to ensure no failure in the wires during the test.

Regarding the total effects of the main groups, results showed that Group 2 (Monobond etch and prime, MEP) recorded statistically insignificant higher retention mean value $(204.63 \pm 35.30 \mathrm{~N})$ than Group 1 (Hydrofluoric acid, HF +silane) mean value $(196.21 \pm 39.76 \mathrm{~N})$. In addition, when the cementation approach was kept constant in both groups, it was found that subgroup 2A reported higher retention mean value $(267.42 \pm 40.92 \mathrm{~N})$ than subgroup 1A $(264.07 \pm 57.78 \mathrm{~N})$. Moreover, subgroup $2 \mathrm{~B}$ recorded higher retention mean value $(141.84 \pm 29.68 \mathrm{~N})$ than subgroup 1B $(128.35 \pm 21.75$ $\mathrm{N})$. Therefore, the first hypothesis which states that the novel one step ceramic surface treatment will give the same results as the conventional treatment technique was accepted.

This coincides with the results of the study done by Tribst et al. 2018 where they revealed that the self-etching ceramic primer provided statistically insignificant higher bond strength values than conventional surface treatment method. It did not show any drawbacks for its use in terms of bond strength and integrity despite the reduced irregularities that are produced in the glass ceramic surface. In addition, it provided reduced steps and shorter application time. They explained that active application of silane that is contained within MEP attributed to the increase in the bond strength as it increases surface energy of the treated ceramic.

In addition, it was found by F. S. F. Siqueira et al.2016 that MEP provided statistically insignificant higher microshear bond strength values than HF acid and Monobond plus. They pointed out that MEP provided an etching pattern sufficient for adhesive interlocking with glass ceramic despite its reduced etching pattern compared to HF acid. Moreover, they reported that HF acid is sensitive in its application to concentration and duration which can lead to overetching and weakening of the ceramic. Therefore, MEP was considered a simplified and reliable method for glass ceramics etching. (Román-Rodríguez et al. 2017) found that HF and silane had statistically insignificant higher shear bond strength than MEP. They showed that MEP can replace conventional surface treatment of silicate ceramics "while maintaining bond strength and reducing toxicity". On the other hand, (Prado et al. 2018) reported that $\mathrm{HF}+$ silane showed statistically significant higher bond strength values than self-etching ceramic primer (MEP). 
Regarding the cementation approaches, results showed that subgroup A (self-etch resin cement, Rely X ultimate) had statistically significant higher retention mean values $(265.75 \pm 49.35 \mathrm{~N})$ than subgroup B (self-adhesive resin cement, Rely X U200) mean value (135.09 $\pm 25.71 \mathrm{~N})$. Within Group 1 , subgroup 1 A demonstrated statistically significant higher mean retention values $(264.07 \pm 57.78 \mathrm{~N})$ than subgroup 1B $(128.35 \pm 21.75 \mathrm{~N})$. Similarly, within Group 2, subgroup 2A demonstrated statistically significant higher retention mean value $(267.42 \pm 40.92 \mathrm{~N})$ than subgroup $2 \mathrm{~B}(141.84 \pm 29.68$ $\mathrm{N})$. As a result, the second hypothesis which states that both cementation approaches will give similar results was rejected.

This coincides with the study conducted by (Rojpaibool and Leevailoj 2017) where they revealed higher bond strength provided by Rely X Ultimate than Rely X U200 to dental tissues. According to the authors, they explained that this was due to the difference in composition and lower penetration power of Rely X U200. Its content of acidic monomers and phosphoric acid ester cannot adequately condition dentin, modify smear layer or infiltrate into the interfibillar spaces and hence weaker bond. In addition, the presence of the universal adhesive which can efficiently condition, prime and bond efficiently to dentinal tissue and hydroxyapatite crystals (through 10-MDP) in addition to modifying smear layer and hybridization causes also the self-etch adhesive to provide greater bond strength and stability. According to this study, it can be figured out that the self-etch resin cement (Rely X Ultimate) is more reliable than the selfadhesive resin cement in terms of bonding to glass ceramics and tooth structure.

The higher retention value of the self-etch resin cement (Rely X Ultimate) compared to the self-adhesive resin cement (Rely X U200) may be attributed to the lower viscosity of Rely X Ultimate which allows for better wettability of the cement to the surface.
When comparing the four subgroups to each other and arranging them in descending order, the highest retention mean value was obtained with subgroup $2 \mathrm{~A}$ followed by $1 \mathrm{~A}$ then $2 \mathrm{~B}$ followed by $1 \mathrm{~B}$ which indicates that the combination of MEP with selfetch resin cement (Rely X Ultimate) is the best for obtaining the highest possible bond strength. The conventional surface treatment (HF+ silane) along with self-adhesive resin cement provided the least bond strength among all subgroups

Observing modes of failure is also an indicative of the bond strength, subgroup 1A (HF + silane with Rely X Ulimate) and 2A ( MEP with Rely X Ultimate) showed cohesive mode of failure within the IPS e.max press crowns which supports the high bond strength obtained with these groups. On the contrary, subgroup 1B (HF + silane with Rely X U200) showed adhesive mode of failure within the cement which supports the finding of the study that self-adhesive resin cements are weaker than selfetch resin cements. One sample of group 2B (MEP with Rely X U200) showed mixed adhesive cohesive mode of failure while the rest of the subgroup showed adhesive mode of failure within the cement which also supports the results when comparing the subgroups $1 \mathrm{~B}$ and $2 \mathrm{~B}$ where subgroup $2 \mathrm{~B}$ had a higher retention mean value than $1 \mathrm{~B}$.

\section{CONCLUSIONS}

\section{Within the limitations of this study, it can be con- cluded that}

1) The self-etching ceramic primer (Monobond etch and prime, MEP) can be strongly recommended and can efficiently replace the conventional surface treatment method (Hydrofluoric acid, $\mathrm{HF}+$ silane coupling agent) for lithium disilicate glass ceramics omitting the use of the hazardous HF acid.

2) Self-etch resin cements provide superior retention values for lithium disilicate crowns compared to the self-adhesive resin cements. 
3) Combining Monobond etch and prime (MEP) as a surface treatment for lithium disilicate glass ceramics with the self-etch resin cement approach provided the highest retention among all subgroups.

4) Combining the conventional surface treatment (Hydrofluoric acid $\mathrm{HF}+$ silane) for lithium disilicate glass ceramics with the self-adhesive resin cement provided the lowest retention among all subgroups.

\section{RECOMMENDATIONS}

1- In vitro studies on the self - etching ceramic primer after thermomechanical fatigue simulate clinical situations are required.

2- Conducting studies on the effect of the selfetching ceramic primer on bond strength of minimally invasive restorations to the tooth substrate.

3- Further studies are required to compare the effect of the self-etching ceramic primer on different and novel glass ceramics introduced to the market.

4- Further in vivo studies on the self-etching ceramic primer are required to test its clinical performance and bond stability in the clinical situation.

\section{REFERENCES}

- Höland, W., Rheinberger, V., Apel, E., van 't Hoen, C., Höland, M., Dommann, A., Graf-Hausner, U. (2006). Clinical applications of glass-ceramics in dentistry. Journal of Materials Science: Materials in Medicine, 17(11), 1037-1042

- The original lithium disilicate press ceramic The legendary press ceramic. (n.d.).

- Zarone, F., Ferrari, M., Mangano, F. G., Leone, R., \& Sorrentino, R. (2016). "Digitally Oriented Materials": Focus on Lithium Disilicate Ceramics. International Journal of Dentistry, 2016, 1-10.

- Ramakrishnaiah, R., Alkheraif, A. A., Divakar, D. D., Matinlinna, J. P., \& Vallittu, P. K. (2016a). The Effect of
Hydrofluoric Acid Etching Duration on the Surface Micromorphology, Roughness, and Wettability of Dental Ceramics. International Journal of Molecular Sciences, 17(6).

- Ramakrishnaiah, R., Alkheraif, A. A., Divakar, D. D., Matinlinna, J. P., \& Vallittu, P. K. (2016b). The effect of hydrofluoric acid etching duration on the surface micromorphology, roughness, and wettability of dental ceramics. International Journal of Molecular Sciences, 17(6).

- $\quad$ Baratto, S. S. P., Spina, D. R. F., Gonzaga, C. C., Cunha, L. F. da, Furuse, A. Y., Baratto Filho, F., ... Correr, G. M. (2015). Silanated Surface Treatment: Effects on the Bond Strength to Lithium Disilicate Glass-Ceramic. Brazilian Dental Journal, 26(5), 474-477.

- Scientific Documentation - PDF. (n.d.). Retrieved March 16,2018

- Moghaddas, M. J., Hossainipour, Z., Majidinia, S., \& Ojrati, N. (2017). Comparison of the shear bond strength of self-adhesive resin cements to enamel and dentin with different protocol of application. Electronic Physician, 9(8), 4985-4991.

- Johnson, G. H., Lepe, X., Patterson, A., \& Schäfer, O. (2014). Simplified cementation of lithium disilicate crowns: Retention with various adhesive resin cement combinations. The Journal of Prosthetic Dentistry, 1-7.

- $\quad$ Brum, R., Mazur, R., Almeida, J., Borges, G., \& Caldas, D. (2011). The influence of surface standardization of lithium disilicate glass ceramic on bond strength to a dual resin cement. Operative Dentistry, 36(5), 478-485.

- $\quad$ Pollington, S. (2011). Novel glass-ceramics for dental restorations. Journal of Contemporary Dental Practice, 12(1), 60-67.

- Stamatacos, C., \& Simon, J. F. (2013). Cementation of indirect restorations: an overview of resin cements. Compendium of Continuing Education in Dentistry (Jamesburg, N.J. : 1995), 34(1).

- Nawrocka, A., \& Łukomska-Szymańska, M. (2019). Extracted human teeth and their utility in dental research Recommendations on proper preservation: A literature review. Dental and Medical Problems, 56(2), 185-190.

- Qamheya AHA, Q. M. and A. V. (2016). Lithium Disilicate Restorations: Overview and A Case Report Citation: Qamheya AHA, Qamheya M and Arisan V. Lithium Disilicate Restorations: Overview and A Case. (January).

- Vivadent, I. (2012). IPS e.max Press- Wissenschaftliche Dokumentation. Ivoclar Vivadent, 1-37. 
- Peixoto, R. T. R. C., Paulinelli, V. M. F., Sander, H. H., Lanza, M. D., Cury, L. A., \& Poletto, L. T. A. (2007). Light transmission through porcelain. Dental Materials, 23(11), $1363-1368$.

- Giraldo, T. C., Villada, V. R., Castillo, M. P., Gomes, O. M. M., Bittencourt, B. F., \& Dominguez, J. A. (2016). Active and passive application of the phosphoric acid on the bond strength of lithium disilicate. Brazilian Dental Journal, 27(1), 90-94.

- Prado, M., Prochnow, C., Marchionatti, A. M. E., Valandro, L. F., Baldissara, P., \& Wandscher, V. F. (2018). Ceramic surface treatment with a single-component primer: Resin adhesion to glass ceramics. Journal of Adhesive Dentistry, 20(2), 99-105.

- Carrilho, E., Cardoso, M., Ferreira, M. M., Marto, C. M., Paula, A., \& Coelho, A. S. (2019). 10-MDP based dental adhesives: Adhesive interface characterization and adhesive stability-A systematic review. Materials, 12(5), 1-18.

- Betancourt, D. E., Baldion, P. A., \& Castellanos, J. E. (2019). Resin-dentin bonding interface: Mechanisms of degradation and strategies for stabilization of the hybrid layer. International Journal of Biomaterials, 2019.

- $\quad$ Siqueira, F. S., Alessi, R. S., Cardenas, A. F., Kose, C., Souza Pinto, S. C., Bandeca, M. C., ... Gomes, J. C. (2016). New Single-bottle Ceramic Primer: 6-month Case Report and Laboratory Performance. The Journal of Contemporary Dental Practice, 17(12), 1033-1039.

- $\quad$ Tribst, J., Anami, L., Özcan, M., Bottino, M., Melo, R., \& Saavedra, G. (2018). Self-etching Primers vs Acid Conditioning: Impact on Bond Strength Between Ceramics and Resin Cement. Operative Dentistry, 16-348-L.

- Rojpaibool, T., \& Leevailoj, C. (2017). Fracture Resistance of Lithium Disilicate Ceramics Bonded to Enamel or Dentin Using Different Resin Cement Types and. 26, 141-149.

- Román-Rodríguez, J. L., Perez-Barquero, J. A., Gonzalez-Angulo, E., Fons-Font, A., \& Bustos-Salvador, J. L. (2017). Bonding to silicate ceramics: Conventional technique compared with a simplified technique. Journal of Clinical and Experimental Dentistry, 9(3), e384-e386. 